\title{
Effects of breathing exercises on lung capacity and muscle activities of elderly smokers
}

\author{
Hyun-Ju Jun, PT, PhD ${ }^{1)}$, KI-Jong KIm, PT, PhD ${ }^{1)^{*}}, \mathrm{~K}_{\mathrm{I}}-\mathrm{Won}_{\mathrm{I}} \mathrm{Na}, \mathrm{PT}, \mathrm{PhD}^{2)}$, \\ Chang-Heon Kim, PT, PhDc ${ }^{3}$ ) \\ 1) Department of Physical Therapy, Cheongam College: 1641 Noksaek-ro, Suncheon-si, Jeollanam-do, \\ Republic of Korea \\ 2) Department of Physical Therapy, Dongshin University, Republic of Korea \\ 3) Department of Physical Therapy, Design Hospital, Republic of Korea
}

\begin{abstract}
Purpose] Elderly smokers have a reduced chest diameter due to weakening of the respiratory muscles, and this results in decreased ventilation, leading to a vicious circle. Therefore, the present study investigated the effects of an intervention program to enhance the pulmonary function and muscle activity of elderly smokers. [Subjects and Methods] Participants were randomly assigned to one of two experimental groups or a control (CG) group. The experimental groups performed exercises three times per week for six weeks, whereas the CG performed no exercises. One of the experimental groups performed a Feedback Breathing Exercise (FBE) for 15 minutes, and the other repeated three sets of Balloon-Blowing Exercises (BBE) with sufficient rest of more than one minute between sets. [Results] In the experimental groups, FVC, FEV1/FVC, PEF and muscle activity of the rectus abdominis significantly improved after four weeks, but no significant differences were observed in FEV1 or VC after six weeks. [Conclusion] The results show that FBE and BBE improved the pulmonary functions of elderly smokers, demonstrating the potential benefits of the development of various training methods using balloons, and group programs, including recreational factors, for increasing respiratory muscles strength.

Key words: Feedback Breathing Training, Balloon Blowing Training, Elderly smokers
\end{abstract}

(This article was submitted Dec. 23, 2015, and was accepted Feb. 15, 2016)

\section{INTRODUCTION}

South Korea's aging society will lead to an increase in the population aged over 65 years ${ }^{1)}$. Elderly persons are vulnerable to risk factors such as chronic diseases, impaired physical condition, and functional limitations, as well as cardiopulmonary dysfunction and muscle weakness. Thus, it is important that the elderly should perform aerobic and strengthening exercises, and to manage the risk of falls in order to ensure their health ${ }^{2}$. Furthermore, the elderly have age-related diseases and reduced of body functions, which are accompanied by changes in posture. Changes in posture during Activities of Daily Living (ADLs) can affect respiratory activities and cause difficulties with physical movement ${ }^{3)}$.

For the elderly, the maintenance of cardiopulmonary function is a fundamental element of ADL. Reduced cardiopulmonary fitness among the elderly has significant effects, through sarcopenia and physical disability due to aging ${ }^{4}$.

Cardiopulmonary function is an important factor for exercise capacity, and pulmonary ventilation and respiratory strengthening can increase exercise capacity by reducing respiratory fatigue ${ }^{5,6}$. If the cardiopulmonary function required for long-term activities can be maintained, elderly persons can continue to participate in indoor activities as well as community activities such as shopping, leisure activities, and walking, which enhance quality of life ${ }^{7}$.

Smoking can damage pulmonary functions and lead to oxygen insufficiency in cells as a result of elevated carbon monoxide in the blood and decreased hemoglobin oxygen-carrying capacity. Additionally, smoking can reduce fitness and the ability

\footnotetext{
*Corresponding author. Ki-Jong Kim (E-mail: kjparadise@hanmail.net)

(C)2016 The Society of Physical Therapy Science. Published by IPEC Inc.

This is an open-access article distributed under the terms of the Creative Commons Attribution Non-Commercial No Derivatives (by-nc-nd) License $<$ http://creativecommons.org/licenses/by-nc-nd/4.0/>.
} 
to perform exercises ${ }^{8)}$. Smoking is also considered to be one of the major causes of death, because the harmful substances contained in just one cigarette can shorten life by 11 minutes ${ }^{9,10)}$. Approximately $90 \%$ of smokers begin smoking before the age of $25^{11}$. Smoking among young people has continued to increase, thereby causing pulmonary function problems at an early age $\mathrm{e}^{12,13)}$.

In order for smokers to increase their intake of air, it is necessary to maintain coordination among the muscles involved in breathing. These muscles increase rib expansion and diaphragm descent during inspiration, thereby increasing air influx into the lungs, and discharging air via relaxation of the respiratory muscles during expiration ${ }^{14)}$.

Weakness of breathing muscles can induce dyspnea and reduce the ability to perform exercises. Thus, therapeutic interventions are needed to improve breathing and exercise ability through enhancement of respiratory muscle functions ${ }^{15,16)}$. Aerobic exercise can increase muscle strength and the endurance of respiratory muscles as well as respiratory functions ${ }^{17)}$.

Elderly smokers undoubtedly suffer reduced respiratory activity. Elderly people also experience a reduction in chest diameter because of the weakening of respiratory muscles, resulting in ventilation decrease, leading to a vicious circle. Therefore, the present study investigated the effects of an intervention program to enhance the pulmonary function and muscle activities of elderly smokers.

\section{SUBJECTS AND METHODS}

The study recruited 30 smokers aged over 65 years of age. Participation was voluntary, and all subjects were recruited from Mokpo, Jeollanam-do. The subjects smoked or had smoked more than 10 cigarettes a day for longer than one year a day; the general characteristics of the subjects are shown in Table 1. Prior to commencement, the study was approved by the Bioethics Committee of Dongshin University (IRB No. 201406-BM-001-02).

The participants were divided into four groups (each $n=10$ ): a control group (CG), a feedback breathing exercise group (FBEG), and a balloon-blowing exercise group (BBEG). Group allocation was random, using three tickets (ticket $1=\mathrm{CG}$, 2=FBEG, 3=BBEG). The CG performed no exercises. The exercise program was conducted three times per week for six weeks, and no exercise was conducted during the final two weeks. Since the study subjects were all elderly, the exercise program included conditioning and sufficient rest. The feedback breathing exercises involved the use of a SPIRO TIGER ${ }^{\circledR}$ for 15 minutes, while the BBEG repeated three sets of balloon-blowing exercises with sufficient rest of more than one minute between sets.

The pulmonary functions of the experimental groups were measured after the four weeks of breathing exercises were completed, and a follow-up test was conducted two weeks later, without further training. Breathing exercises were conducted while taking care to avoid heart pain and dizziness.

Pulmonary function was measured using a Cardio Touch 3000S (Bionet Co., Ltd., South Korea), while subjects were seated in a chair. The pulmonary functions measured were: forced vital capacity (FVC), forced expiratory volume in one second (FEV1), FEV1/FVC, peak expiratory flow (PEF), and vital capacity (VC). FVC, FEV1, and FEV1/FVC are used to determine obstructive and restrictive lung diseases; and PEF is used to determine airway resistance.

To ensure accurate and reliable data, the pulmonary functions were measured in triplicate with the understanding and cooperation of the subjects with the method, and the highest values were selected ${ }^{18)}$.

Surface EMG measurements were made using a POCKET EMG (BTS Co., Italy) of muscles related to breathing on the left and right sides of the rectus abdominis via four channels. During deep breathing, three muscle activity measurements were conducted with a one-minute rest between measurements, and the mean value was calculated.

Statistical processing employed Predictive Analytics Software (PASW, version 18.0). Subjects' general characteristics were analyzed using one-way analysis of variance (ANOVA). Significance effects of time within a group were tested using repeated-measures ANOVA, and the Bonferroni test was conducted as a the post hoc test. If there was a significant effect of time within a group a repeated contrast test was conducted for each group. In all tests, the significance level was 0.05 .

\section{RESULTS}

Repeated-measures ANOVA showed that, in the FBEG group, FVC, FEV1/FVC, PEF, and muscle activity in the rectus abdominis had increased significantly after four weeks $(\mathrm{p}<0.05)$, but reduced significantly in the following two weeks $(\mathrm{p}<0.05)$. VC had significantly increased after four weeks $(\mathrm{p}<0.05)$ but no significant difference was found after six weeks $(\mathrm{p}>0.05)$.

In the BBEG group, FVC, FEV1/FVC, PEF, and muscle activity in the rectus abdominis had increased significantly after four weeks $(\mathrm{p}<0.05)$, but reduced significantly in the following two weeks $(\mathrm{p}<0.05)$. FEV1 and $\mathrm{VC}$ had increased significantly after four weeks $(p<0.05)$, but no significant differences were found after six weeks $(p>0.05)($ Table 2$)$.

\section{DISCUSSION}

In the FBEG group, FVC, FEV1/FVC, PEF, VC, and rectus abdominis had significantly increased after 4 weeks, but the increase was not maintained in VC after 6 weeks. In the BBEG group, FVC, FEV1, FEV1/FVC, PEF, VC, and rectus 
Table 1. General characteristics of the subjects

\begin{tabular}{lccc}
\hline & CG $(\mathrm{n}=10)$ & FBEG $(\mathrm{n}=10)$ & BBEG $(\mathrm{n}=10)$ \\
\hline Gender (male/female) & $7 / 8$ & $8 / 2$ & $7 / 3$ \\
Age (years) & $78.3 \pm 5.7$ & $72.6 \pm 9.0$ & $70.0 \pm 3.5$ \\
Height $(\mathrm{cm})$ & $167.2 \pm 5.6$ & $163.2 \pm 6.4$ & $152.7 \pm 6.3$ \\
Weight $(\mathrm{kg})$ & $63.2 \pm 9.4$ & $66.6 \pm 9.9$ & $59.5 \pm 9.6$ \\
\hline
\end{tabular}

Values are mean \pm SD. CG: control group; FBEG: feedback breathing exercise group; BBEG: balloon-blowing exercise group

Table 2. Changes of pulmonary function and muscle activity of the groups

\begin{tabular}{llcccc}
\hline Pulmonary function & $\begin{array}{l}\text { Experimental } \\
\text { groups }\end{array}$ & Pre & 2 weeks & 4 weeks & 6 weeks \\
\hline & CG & $3.23 \pm 0.28$ & $3.23 \pm 0.27$ & $3.23 \pm 0.27$ & $3.23 \pm 0.27$ \\
FVC (L) & FBEG $\dagger$ & $3.40 \pm 0.18$ & $3.44 \pm 0.24$ & $3.77 \pm 0.30^{*}$ & $3.66 \pm 0.20^{*}$ \\
& BBEG $\dagger$ & $3.26 \pm 0.33$ & $3.46 \pm 0.61$ & $3.89 \pm 0.50^{*}$ & $3.64 \pm 0.41^{*}$ \\
& CG & $2.47 \pm 0.53$ & $2.48 \pm 0.52$ & $2.48 \pm 0.51$ & $2.48 \pm 0.51$ \\
FEV1 (L) & FBEG & $2.68 \pm 0.54$ & $2.70 \pm 0.55$ & $2.80 \pm 0.61$ & $2.71 \pm 0.52$ \\
& BBEG & $2.54 \pm 0.60$ & $2.69 \pm 0.46$ & $3.13 \pm 0.54^{*}$ & $2.78 \pm 0.47$ \\
FEV1/FVC & CG & $69.40 \pm 10.78$ & $69.60 \pm 11.26$ & $69.60 \pm 10.77$ & $69.40 \pm 11.37$ \\
$(\%)$ & FBEG & $69.90 \pm 9.50$ & $69.90 \pm 9.80$ & $74.10 \pm 9.35^{*}$ & $69.90 \pm 8.97^{*}$ \\
& BBEG & $73.00 \pm 9.07$ & $73.10 \pm 9.50$ & $76.10 \pm 9.61^{*}$ & $73.30 \pm 10.50^{*}$ \\
PEF (L) & CG & $3.69 \pm 1.10$ & $3.68 \pm 1.10$ & $3.69 \pm 1.08$ & $3.69 \pm 1.08$ \\
& FBEG & $4.20 \pm 1.38$ & $4.20 \pm 1.39$ & $4.23 \pm 1.39^{*}$ & $4.20 \pm 1.39^{*}$ \\
VC (L) & BBEG & $3.40 \pm 1.16$ & $3.40 \pm 1.19$ & $3.64 \pm 1.24^{*}$ & $3.53 \pm 1.23^{*}$ \\
& CG & $2.81 \pm 0.07$ & $2.80 \pm 0.07$ & $2.80 \pm 0.07$ & $2.79 \pm 0.08$ \\
Rectus abdominis & FBEG & $2.95 \pm 0.43$ & $2.95 \pm 0.43$ & $3.32 \pm 0.49^{*}$ & $3.22 \pm 0.51$ \\
$(\%)$ & BBEG & $2.98 \pm 0.37$ & $2.99 \pm 0.37$ & $3.31 \pm 0.30^{*}$ & $3.23 \pm 0.40$ \\
& FBEG & $0.35 \pm 0.10$ & $0.35 \pm 0.10$ & $0.35 \pm 0.10$ & $0.35 \pm 0.10$ \\
\hline
\end{tabular}

Mean \pm SD. *Significant difference compared with Pre $(\mathrm{p}<0.05)$. †Significant difference compared with $C G(p<0.05) . C G$ : control group; FBEG: feedback breathing exercise group; BBEG: balloon-blowing exercise group

abdominis had significantly increased after 4 weeks, but the increases were not maintained in FEV1 and VC after 6 weeks. As a result of medical advances and economic development, the elderly population has been increasing, and in tandem with this the number of respiratory patients, such as those with chronic obstructive pulmonary disease (COPD) has also been increasing ${ }^{19,20)}$. In a previous study, respiratory physical therapy was conducted for 40 minutes five times per week for four weeks for stroke patients aged around 60, and it elicited increases in $\mathrm{FVC}^{21)}$. This result was similar to the finding of the present study, in which the FBEG and BBEG groups of elderly smokers showed significant increases in pulmonary functions after four weeks of exercise. The effects of breathing exercises performed by stroke patients and the elderly have been reported in previous studies; however, the present study is more meaningful, because our subjects were older. Nevertheless, despite the significant benefits observed at the end of the four-week exercise program, the FBEG and BBEG groups showed significantly reduced pulmonary function after the two-week period of no intervention. This indicates that for lasting improvement, exercise is required for a period longer than four weeks.

It was previously reported that, following endurance training of the respiratory muscles of COPD and spinal cord injury patients, FEV1/FVC increased significantly ${ }^{22)}$. A study of pulmonary functions of spinal cord injury patients, measured during inpatient rehabilitation and following hospital discharge, showed that only FEV1/FVC increased during the inpatient rehabilitation period ${ }^{23)}$. This result is similar to the finding of the present study, in which the FBEG and BBEG groups of elderly smokers showed significant increases in pulmonary function after four weeks, but significant decreases after no intervention during the subsequent two weeks. In summary, breathing exercises are effective for COPD patients, spinal cord injury patients, and the elderly. Therefore, it is necessary to study exercise methods that can continuously maintain the effects of breathing exercises.

Measurement of PEF is a method of evaluating the level of peak airway obstruction. PEF significantly increased in chronic 
stroke patients who performed FBE three times per week for eight weeks ${ }^{24)}$. In the present study of elderly smokers, PEF also increased significantly after four weeks of FBE and BBE. This result indicates that the effects of exercise were apparent earlier than in the above-noted study of breathing exercises, conducted over 8 weeks with chronic stroke patients.

Intervention programs should last a minimum of 12 weeks in order to see effects on physical activities with respect to respiratory functions, including cardiovascular functions ${ }^{25}$. In the present study, the beneficial effects of the intervention training were observed after four weeks, but declined significantly once the intervention was stopped. If the interventions had continued for more than 12 weeks, the effect may have been maintained.

It was previously reported that breathing training for stroke patients induced diaphragm movements and contractions in the internal intercostalis muscles as a result of active and strong contractions in the rectus abdominis, thereby further facilitating respiratory activity and increasing respiratory muscle activities ${ }^{24)}$. In a study of subjects with spinal cord injuries, electrical stimulation was applied to the rectus abdominis and muscles around the abdomen, and it improved and tidal volume and pulmonary ventilation ${ }^{26}$. In the present study, increases in the muscle activity of the rectus abdominis an accessory muscle of respiration occurred and accessory muscles affect the diaphragm, thereby influencing the internal intercostalis, which is a respiratory muscles. The increased in rectus abdominis activity supports the measurement of muscle activity in the rectus abdominis as a means of examining the effects of breathing exercises on pulmonary function. In a study of patients with lower back pain, muscle activities of the abdominal obliques, erector spinae, and rectus abdominis increased following abdominal breathing exercises conducted five times per week for 6 weeks ${ }^{27}$. These results show that breathing exercises not only strengthen the rectus abdominis, but also improve trunk stability by strengthening the erector spinae, which is an antagonist muscle of the trunk. On the basis of the results cited above, breathing training for elderly patients increases the muscle activity of the rectus abdominis, thereby increasing core stability and reducing the risk of falls, improving their quality of life.

Inspiration in the normal state expands the rib cage above and below the rib portions as a result of contraction of the diaphragm and other respiratory muscles. This is a result of reduced pressure inside the chest, followed by the promotion of inspiration ${ }^{28)}$. Respiratory exercise can induce contraction of the muscles involved in breathing, including the intercostalis and the rectus abdominis, leading to increased activity among respiratory muscles.

The FBE program utilized in the present study can train inspiration and expiration muscles, and the BBE exercise of inflating a balloon can adjust the volume of expiration after strong inhalation followed by exhalation. Diaphragm respiratory exercises significantly increased tidal volume, inspiratory reserve volume, inspiratory capacity, and breathing capacity of long-term male smokers ${ }^{29}$. This increase in six-minute walking distance would increase the ability to engage in everyday activities, thereby having a positive effect on quality of life.

A limitation of this study was that the ratio of male to female subjects was very large, because of the social characteristics of smoking. It also did not set a criterion for daily smoking amount and did not analyze subjects who had smoked for more than 65 years, or their length of time as smokers. Future research should address these limitations.

This study showed that FBE and BBE increased the pulmonary function of elderly smokers. The BBE results show that pulmonary function can be improved without the use of specialist equipment and that the use of balloons, which can be easily purchased, regardless of location, had a positive effect. Thus, we emphasize the need for the development of various training methods using balloons, and the importance of group program development, including recreational factors, to increase the function of respiratory muscles.

\section{REFERENCES}

1) Kim SK: Effect of Aerobic Exercise on the Improvement in Middle Aged Women. Master's thesis, Graduate School of Korea National Sport University, 2008, pp 1-3.

2) Nelson ME, Rejeski WJ, Blair SN, et al. American College of Sports Medicine American Heart Association: Physical activity and public health in older adults: recommendation from the American College of Sports Medicine and the American Heart Association. Circulation, 2007, 116: 1094-1105. [Medline] [CrossRef]

3) Mori RL, Bergsman AE, Holmes MJ, et al.: Role of the medial medullary reticular formation in relaying vestibular signals to the diaphragm and abdominal muscles. Brain Res, 2001, 902: 82-91. [Medline] [CrossRef]

4) Chien MY, Kuo HK, Wu YT: Sarcopenia, cardiopulmonary fitness, and physical disability in community-dwelling elderly people. Phys Ther, 2010, 90: 1277-1287. [Medline] [CrossRef]

5) Jung HJ, Lee DT: Inspiratory muscle training, aerobic exercise, pulmonary function, maximal oxygen uptake, inspiratory pressure. Exerc Sci, 2012, 21: 373-384. [CrossRef]

6) Roh H, Lee D, Lee S, et al.: Respiratory muscle training of pulmonary function for smokers and non-smokers. J Phys Ther Sci, 2012, 24: 691-693. [CrossRef]

7) Byun YH, Kim SH, Kim TS: Effect of body mass index difference on body composition, cardiopulmonary, low extremity muscular functions in institutionalized older women. J Sport Leis Stud, 2013, 52: 789-799.

8) Cha KS: A comparison of pulmonary function, aerobic power, blood pressure, blood hemoglobin in smokers and non-smokers. Korean J Phys Educ, 2001, 40: 845-857.

9) Mokdad AH, Marks JS, Stroup DF, et al.: Actual causes of death in the United States, 2000. JAMA, 2004, 291: 1238-1245. [Medline] [CrossRef]

10) Shaw M, Mitchell R, Dorling D: Time for a smoke? One cigarette reduces your life by 11 minutes. BMJ, 2000, 320: 53-53. [Medline] [CrossRef]

11) Kim J, Lim SA: The realities of smoking among some college students and their relevant knowledge. J Korean Soc Dent Hyg, 2006,6 : 283-294. 
12) Tantisuwat A, Thaveeratitham P: Effects of smoking on chest expansion, lung function, and respiratory muscle strength of youths. J Phys Ther Sci, 2014, 26: 167-170. [Medline] [CrossRef]

13) Lee SK, Park JW, Kim KH, et al.: An analysis of the thickness of abdominal muscles during forceful expiration and pulmonary function in teenage smokers and nonsmokers. J Phys Ther Sci, 2013, 25: 789-791. [Medline] [CrossRef]

14) Kim JS: Effects of a balloon-blowing exercise on lung function of young adult smokers. J Phys Ther Sci, 2012, 24: 531-534. [CrossRef]

15) Weiner P, Magadle R, Beckerman M, et al.: Comparison of specific expiratory, inspiratory, and combined muscle training programs in COPD. Chest, 2003, 124: 1357-1364. [Medline] [CrossRef]

16) Kido S, Nakajima Y, Miyasaka T, et al.: Effects of combined training with breathing resistance and sustained physical exertion to improve endurance capacity and respiratory muscle function in healthy young adults. J Phys Ther Sci, 2013, 25: 605-610. [Medline] [CrossRef]

17) Carr M, Jones J: Physiological effects of exercise on stroke survivors. Top Stroke Rehabil, 2003, 9: 57-64. [Medline] [CrossRef]

18) Kim DS: The relationship of age, weight, height and smoking history with spirometric parameters in healthy adults. Bulleum Dongnam Health Coll, 2003, 21: 429-440.

19) Kim YS: The effect of the combined exercise on the risk factors for health related physical fitness, blood lipids, and cardiovascular diseases in the elderly women. Doctorial thesis, Graduate School of Kangnung National University, 2007, pp 9-11.

20) Na HR: Explorative study on fatigue in patients with chronic obstructive pulmonary disease. Master's thesis, Graduate School of Kyung Hee University, 2000, pp 7-9.

21) Kim JH, Hong WS, Bae SS: The effect of chest physical therapy on improvement of pulmonary function in the patients with stroke. J Kor Soc Phys Ther, 2000, 12: $133-144$.

22) Scherer TA, Spengler CM, Owassapian D, et al.: Respiratory muscle endurance training in chronic obstructive pulmonary disease: impact on exercise capacity, dyspnea, and quality of life. Am J Respir Crit Care Med, 2000, 162: 1709-1714. [Medline] [CrossRef]

23) Mueller G, de Groot S, van der Woude L, et al.: Time-courses of lung function and respiratory muscle pressure generating capacity after spinal cord injury: a prospective cohort study. J Rehabil Med, 2008, 40: 269-276. [Medline] [CrossRef]

24) Seo KC: The Effect of Pulmonary Function and Respiratory Muscle Activity in the Stroke Patients after Complex Breathing Exercise. Doctorial thesis, Graduate School of Daegu University, 2012, pp 16-20.

25) Song RY, Park IS, So HY, et al.: Applicability and program effects of tai chi exercise in outpatients with coronary artery disease. Korean J Adult Nurs, 2008, 20: $537-547$.

26) Kandare F, Exner G, Jeraj J, et al.: Breathing induced by abdominal muscle stimulation in individuals without spontaneous ventilation. Neuromodulation, 2002, 5: 180-185. [Medline] [CrossRef]

27) Kim K, Park RJ, Bae SS: Effect of diaphragmatic breathing exercise on Activation of trunk muscle of patients with low back pain. J Kor Soc Phys Ther, 2005, 17: $311-327$.

28) Cahalin LP, Braga M, Matsuo Y, et al.: Efficacy of diaphragmatic breathing in persons with chronic obstructive pulmonary disease: a review of the literature. J Cardiopulm Rehabil, 2002, 22: 7-21. [Medline] [CrossRef]

29) Seo K, Park SH, Park K: Effects of diaphragm respiration exercise on pulmonary function of male smokers in their twenties. J Phys Ther Sci, 2015, 27: 2313-2315. [Medline] [CrossRef] 